19

\title{
Approaches to Community Consultation in Exception from Informed Consent: Analysis of Scope, Efficiency, and Cost at Two Centers
}

Louis Eubank ${ }^{1}$, Kwan S. Lee ${ }^{2}$, David B. Seder ${ }^{1}$, Tania Strout ${ }^{1}$, Matthew Darrow ${ }^{2}$, Catherine McDonald ${ }^{2}$, Teresa May ${ }^{1}$, Richard R. Riker ${ }^{1}$, and Karl B. Kern ${ }^{2}$

1. Departments of Critical Care Services and Emergency Medicine, Maine Medical Center, Portland, Maine

2. Division of Cardiology, University of Arizona, Sarver Heart Center, Tucson, Arizona

Total Words: 3019

Keywords: Exception; Consent; EFIC, Community; Consultation; Research

Corresponding author:

Karl B. Kern, MD

Professor of Medicine

The Gordon A. Ewy, M.D. Distinguished Endowed Chair of Cardiovascular Medicine Sarver Heart Center

University of Arizona

1501 N. Campbell Avenue

Tucson, Arizona 85724

USA 
Abstract

Objectives: Community consultation (CC) is fundamental to the Exception from Informed Consent (EFIC) process for emergency research, designed to inform and receive feedback from the target study population about potential risks and benefits. To better understand the effectiveness of different techniques for $\mathrm{CC}$, we evaluated EFIC processes at two centers participating in a trial of early cardiac catheterization following out-of-hospital cardiac arrest.

Methods: We studied the Institutional Review Board-approved CC activities at Maine Medical Center (MMC) and University of Arizona (AZ) in support of NCT02387398. In Maine, the public was consulted by survey at a professional basketball game and in the emergency department waiting room (in-person group), by multimedia direction to an online website (online group), and by mail (mailing group). Arizona respondents were either approached at a county fair (in-person group) or were directed to an online survey (online group) via social media advertising.

Results: Among 2185 survey respondents, approval rates were high for community involvement and personal participation without individual consent. Community consultation using in-person, online, and mailed surveys offered slightly different approval rates, and the rate of responses by modality differed by age and education level but not ethnicity. Print advertising was the least cost effective at

$51 \$ 442$ per completed survey.

Conclusions: Canvassing at public events was the most efficient mode of performing CC, with approval rates similar to mailings, online surveys, and canvassing in other locations. Print advertisements in local papers had a low yield and cost more than other approaches. 


\section{Introduction}

Informed consent is considered the bedrock of medical research ${ }^{1}$, yet emergency research is challenging to conduct under traditional informed consent due to a patient's decreased level of consciousness and the absence of an appropriate surrogate decision maker. This is particularly true in cardiac arrest, stroke, and trauma - each of which has often been described as a public health crisis in need of ongoing research into new treatments and therapeutic approaches ${ }^{2-4}$. This conundrum led the Food and Drug Administration to develop and in 1996 enact Regulation 21 CFR 50.24: Exception from Informed Consent (EFIC) Requirements for Emergency Research. EFIC is intended to allow for medical research in circumstances of grave need when true informed consent cannot be obtained. It functions by allowing such research after community dialogue, IRB approval to proceed, with attempted individual consent post enrollment. Specifics as to how community consultation should be performed have been left vague to allow for interpretation by local Institutional Review Boards (IRBs), the evolution of standard practices, and development of precedent.

There are no guidelines regarding the modalities employed and extent of the community consultation that must be performed in EFIC, including the number of surveys performed, need for focus groups and interviews, or required percentages of community members who must agree with and accept the research in a general sense or in terms of their own participation. The FDA addressed this in the addendum guidelines to 21 CFR 50:24: "How much community consultation activity is necessary?

There is no single acceptable way to accomplish or fulfill the community consultation requirements, nor will all studies require the same amount, type, or extent of community consultation activities." 5 This puts investigators and IRBs in the position of developing their own process for each trial, and continuously defining standards of acceptance, which can be quite variable from community to community. For example, one review of nine IRB-approved EFIC trials ${ }^{6}$ found that surveyed community members' personal acceptance rates (the respondent theoretically accepts the possibility of 
79 being enrolled in the trial without consent if they fall ill) varied from $45 \%$ of the survey population to $8093 \%$, with a mean of $68 \%( \pm 12.9 \%, \mathrm{n}=9,036)$. Community acceptances (the respondent theoretically

81 accepts that the research trial be conducted in their community) ranged from $74 \%$ to $100 \%$ with a 82 mean of $78 \%(\mathrm{SD} 10.3 \%, \mathrm{n}=3,797)$. Within these studies, community consultation involved such 83 methods as administering surveys at a state fair, community meetings or other local events, online 84 surveys, telephone surveys via random digit dialing, in-person interviews, and focus groups. Whether 85 these methods yield similar or adequate results is largely unknown, and the labor and expense 86 involved varies greatly, making budgetary planning for EFIC trials difficult.

87 This project is an analysis of the effectiveness and cost of two EFIC community consent 88 processes related to the randomized Pilot Clinical Trial for Early Coronary Angiography Versus No 89 Early Coronary Angiography for Post-Cardiac ARrest Patient with No ST Segment ELevation on their 90 ECG (PEARL) trial (NCT02387398) at University of Arizona, Tucson, Arizona (Arizona) and Maine 91 Medical Center, Portland, Maine (Maine). 


\section{Methods}

\section{Study Design}

Both UAZ and MMC received IRB approval - first to conduct their proposed community consultation, the results of which were later considered in the final IRB approval to conduct the trial under EFIC regulations. There were differences in what each IRB required for community consultation - the University of Arizona, approved a plan of in-person canvassing at a County Fair, supplemented by online surveys and print newspaper advertisements. At Maine Medical Center, the IRB requested diverse community consultation in the form of presentation at community meetings, online surveys, mailed surveys, and canvassing in multiple locations.

Both medical centers informed and surveyed adult community members about the proposed clinical trial, assessing whether respondents thought such a trial was warranted, and the consent mechanism adequate (see Appendix 1 for the survey). Including respondent's zip codes in the survey helped to confirm that many of them lived in the geographic catchment areas of the participating medical centers. The survey also provided respondents the opportunity to put their names on an "opt out" list. Aside from an oral presentation to the Portland City Council, the administration of these surveys was the primary means of eliciting feedback and quantitating approval from the communities. Random number dialing was not part of either EFIC plan due to its expense. ${ }^{12,13}$

\section{University of Arizona}

\section{Online process}

Facebook was the main online tool for alerting the public of this potential study in their community and eliciting survey responses. Twitter was also utilized in this effort.

\section{Print Media Advertisements}


Regional and local newspapers, including two Spanish language papers, were paid to run

118

119

120

121

122

123

124

125

126

127

128

129

130

131

132

133

134

135

136

137

138

advertisements. They described the PEARL study and directed potential respondents to the Facebook survey site.

\section{Direct administration of surveys}

A booth was obtained at the Pima County Fair where compression-only cardiopulmonary

resuscitation was being taught to interested fair-goers. Participants and spectators were then asked if they would be willing to participate in a survey about an upcoming trial at the Arizona Heart Center. Surveys were administered in-person by research staff and investigators (Spanish interpreters were available).

\section{Maine Medical Center}

Online process

Public disclosures and advertisements directed community members to an online web page and survey site. Flyers were posted at the town halls \& libraries in twenty towns and cities within the MMC patient catchment area. Advertisements directing the public to the survey web page were posted on the hospital's Facebook page. Public Service Announcements were made on local radio stations, and print advertisements were placed in local area newspapers directing interested individuals to go to the survey website, or to call and speak to research staff.

Direct administration of surveys

Surveys were administered in-person by research staff and investigators at a local professional basketball game and in Maine Medical Center's Emergency Department waiting room.

Targeted mailing of surveys 
Six thousand, eight hundred letters were mailed to active patients of the primary local

140 Cardiology practice. The mailing included a survey, a self-addressed stamped envelope, and the

141 research office telephone number for direct assistance in interpreting and completing the survey.

142 Completed surveys were returned by mail.

143

144

145

146

147

148

149

150

151

152

153

154

155

156

157

158

159

160

161

\section{Human Subjects}

Institutional Review Boards (IRBs) at UAZ and MMC approved the local PEARL community consultation plans. The protocol at both institutions (and general protocol for conducting EFIC research across most IRBs in the United States) includes an initial presentation wherein the IRB is informed of the research, especially the justification for performing such research under "Exception From Informed Consent" regulations, and the planned community consultation process. The researchers then conducted community consultation and reported their findings to the IRB prior to final approval of the trial.

\section{Statistical Methods}

Survey and cost data were entered into a Microsoft Excel (Microsoft Corp., Redmond, WA) spreadsheet program for cleaning and were analyzed using SPSS v. 24.0 for Windows statistical software (SPSS, Inc., Chicago, IL). No formal strategy was used to the sample size for the survey. We used a convenience sample of individuals willing to participate. We note that previously acceptable community surveys averaged 500 individuals (NIH ROC EFIC studies). To maximize sample size, missing data were excluded on a case-by-case basis. Descriptive statistics were employed to evaluate the characteristics of the study participants and are reported as numbers and percentages. Categorical comparisons were made using Pearson's chi-square or Fisher's exact test, as appropriate. Racial and 
162 ethnic minorities were collapsed into dichotomous categories (White, non-White; Hispanic/Latino, 163 non-Hispanic/Latino) due to small numbers. Education level was similarly collapsed (less than high

164 school, high school/equivalency degree or higher). For continuous data, comparisons were made using 165 the independent samples $t$-test or analysis of variance, as appropriate for the number of groups being 166 evaluated. Spearman's rho was employed to examine the relationships between categorical variables.

167 For the binary outcome variables representing personal and community acceptance of the EFIC trial, 168 logistic regression analyses were conducted to identify participant characteristics associated with EFIC 169 acceptance. We accepted a 2-tailed alpha of $\leq 0.05$ as significant and computed $95 \%$ confidence 170 intervals using the exact method. To adjust for multiple comparisons, Bonferroni's correction was 171 applied. 


\section{Results}

Demographic characteristics of survey participants are provided in Table $\mathbf{1 .}$

\section{Demographic Differences amongst Survey Modalities and Comparisons to County Demographics}

There were differences between the age of the study participants, the racial composition of the sample, reported ethnic identity, and education level when compared to available local demographic characteristics for the relevant counties ( $p$-value range: $0.0404-<0.0001)$ (see Table 2). Maine respondents were older, less racially and ethnically diverse, and more educated than the county-atlarge. Arizona participants were significantly younger and also better educated than their respective county demographics. Within the site-specific modalities, in-person survey response groups most closely approximated county demographics.

In the Maine cohort, the 60+ age group (vs. the 18-59 year age group) was better represented by mail participants (established patients of the local cardiology groups) than in-person and online modalities ( $85 \%$ mail, $27 \%$ in person, $27 \%$ online; $\chi^{2}=470.358, d f=2, p<0.001$ ). In addition, the proportion of participants with at least a high school diploma was greater with the online modality $(100 \%)$ compared to the in-person (96\%) and mailing $(95 \%)$ methodologies $\left(\chi^{2}=12.9, d f=2, p=\right.$ $0.0016)$.

\section{EFIC Acceptance and Differences by Survey Modality}

Across participants, the majority favored the conduct of EFIC research in their communities (2017/2185, 92\%) These data are shown in Figure 1). Younger age correlated with personal acceptance for the trial $(\rho=0.131, p<0.001)$. In addition, higher educational level was associated with EFIC participation $(\rho=-0.109, p<0.001)$. Race and ethnicity were not independently associated with support for EFIC in the community. 


\section{Cost-benefit analysis of various modalities}

196 Estimated costs of soliciting and receiving EFIC feedback through different modalities are

197 shown in Table 3 and Figure 2. Financial costs refer to direct expenditures only. Estimates of hours

198 spent vary by modality - online and mailing groups are an aggregate of time over multiple weeks while

199 the in-person group is time actively spent administering surveys.

200 


\section{Discussion:}

202

The IRB-approved methodologies differed significantly at two large, regional referral centers conducting emergency research under the Exception from Informed Consent (EFIC) rubric.

Community consultation was performed largely by surveys, and respondents to surveys differed demographically from the local population in age, race, ethnicity, and educational level. In-person surveys produced the closest matches to the demographics of their county and were the least expensive and most time-efficient way to perform EFIC consultation. Younger respondents were more likely to respond to online surveys and to approve of EFIC research than older ones, who responded more often by mail than online. Study approval did not vary by race or ethnicity. Online and in-person surveys performed in hospital waiting rooms were the most time-consuming way to obtain community feedback per response obtained. In terms of "hard costs" for advertising and mailings, directing respondents to online surveys by print, radio, and online advertisements cost twice as much per response as the mailings, while in-person surveying at large events carried minimal direct expenses. These data may be helpful for clinical researchers and Institutional Review Boards planning clinical trials that will employ the EFIC process.

Most published EFIC studies have utilized community meetings, random-digit-dialing, and/or surveys for community consent. Community meetings about the specific trial facilitate longer and more involved discussions with community members, and dissemination of complicated information to a number of people at one time. Unfortunately, the reported experience with this approach is uniformly poor. ${ }^{7-9}$ Attendance is typically low (often less than 25 individuals), and the feedback and support obtained may not be representative of the study population. These biases were supported anecdotally by our experiences. 
In contrast, presenting the proposed trial at existing community events outside the hospital

224 offers researchers a unique opportunity to engage the community. Though attendance was small when

225 our investigators presented to the Portland, Maine city council, they were able to speak to critical

226 constituent stakeholders including city counselors, media, and the Fire and Police Chiefs, all of whom

227 strongly supported the trial. This approval is especially important when one considers the likelihood of

228 media coverage when EFIC trials are reported, and the importance of logistical support from civic

229 leaders. $^{10}$

Prior studies have found random-digit-dialing is more successful than community meetings response rates of almost $50 \%$ have occasionally been reported. ${ }^{11}$ However, there are a concerns about sampling error given increasing variability in landlines in peoples' homes., and the increasing adoption of "no call" lists ${ }^{12,13}$ In addition, there may not be a professional surveying company available, capable, or willing, as was the case with Maine, and may often be the case when a research question is complex. Even if said company is available, the cost may be prohibitive: in Arizona a company estimated $\$ 30,000$ for 500 completed PEARL surveys. This would have made random-digit-dialing

237 four times as expensive per survey as any other approach.

Surveys, both online and in person, have been included in many EFIC trials, and compare

239 favorably to community meetings and random-digit-dialing ${ }^{6-11,14-21}$. Survey data exist in the literature

240 for comparison, its costs are substantially lower, and the public is more likely to participate since it 241 takes less time than a community meeting ${ }^{14}$ and is less personally disruptive than random digit dialing.

242 Our community approval data echo the heterogeneity of other published studies of community 243 consent. In fourteen prior studies, eight ${ }^{7,15-21}$ showed no relationship of age or educational level to 244 study approval, however three ${ }^{7,15,21}$ did note racial differences in EFIC approval. Two trials ${ }^{11,22}$ 245 showed greater EFIC acceptance among younger and more educated patients while two other trials ${ }^{23,24}$ 
246 showed increased acceptance with increasing age, one ${ }^{25}$ showed increased acceptance with less

247 education, and one ${ }^{26}$ showed increased acceptance with middle age, and less acceptance in the young

248 and elderly groups as well as a racial difference in acceptance.

249 Online surveys

250 Soliciting and obtaining an online group of respondents was the most expensive method of 251 community consent at $\$ 15.63$ per completed survey and was not time-efficient. However, directing 252 respondents to online surveys offers the advantages of being able to post a considerable amount of 253 information about the specific trial and EFIC in general in the website, which could be further 254 stratified by reading level or interest. On-line surveys can continue during the trial and provide on255 going feedback from the community and patient base. An additional benefit of the information is that 256 both the IRB and researchers can be assured that there is at least one public location where factual data 257 are available - especially important if local media depict EFIC trials in a negative light or report 258 inaccurately. ${ }^{10}$ Finally, online surveys offer access to individuals who are homebound for medical or 259 social reasons, increasing the diversity of respondents to the community consultation process. Given 260 these benefits we believe online consultation to be useful, and a better-calibrated use of advertising on 261 social media might significantly reduce the cost per survey ${ }^{8,28}$. Survey Mailing

The directed survey mailing brought in the greatest overall number of respondents, many of whom were potential study participants, but also generated many telephone calls from confused

265 patients who could not understand the survey materials without coaching from the research staff. This 266 highlighted how face-to-face surveying allowed respondents to get answers to their questions 267 immediately. In the mailed surveys, respondents sometimes wrote in questions, provided comments 268 that were difficult to interpret, or did not fill in all areas of the survey correctly, necessitating still more 
269 phone calls from the research staff. These issues made the mailing of surveys useful, but time-

270 consuming and expensive. Nonetheless, such responses by study staff provide an important avenue of

271 continued dialogue and effort to reach out to the community where such EFIC trials are being

272 conducted.

\section{Limitations}

There are limitations to our study. Although the number of community members consulted was

275 high and approval levels in our samples were significantly higher than many prior EFIC trials, in each

276 case the number was still less than $1 \%$ of the county population, raising the question of whether the

277 sample size was adequate. Community consultation, however, was never intended achieve perfect

278 acceptance $^{29}$ nor to be an exhaustive survey of a community's population ${ }^{30}$. We believe, and our IRBs

279 concurred, that this sampling reflected an adequate community consultation, and showed an

280 appropriate level of acceptance of the trial.

281 Although the Maine and Arizona survey populations are consistent with their respective county

282 demographics, our findings may not be generalizable to other racial and ethnic communities, including

283 Native Americans in Arizona and African refugees in Maine. Additionally, as an editorial to a

284 previous trial mentioned, ${ }^{31}$ there is a concern that the survey text is poorly understood by respondents,

285 some of whom wrote comments such as “...definitely enroll me, I want the best chance to survive!”.

286 However, we feel that these comments could be construed as less about therapeutic misconception

287 than difficulty comprehending equipoise and randomization ${ }^{32}$. This is borne out by a number of people

288 writing comments such as "I don't care which group I'm in, I want to be in the one that has the best

289 survival" and "Why randomize me? Just put me in the one that will give me the best chance of

290 success!" 
The PEARL trial is a comparatively small extension of current best practice (catheterization of those patients with STEMI post-ROSC). Therefore, our data may not be applicable to EFIC trials that

293 have a relatively larger deviation from best practice, employ more invasive or experimental therapies, 294 or are targeted at vulnerable populations. Finally, this study may have little to no generalizability for 295 neurologic or trauma studies, as there have been no studies to date looking at EFIC acceptance 296 stratified by etiology of injury.

297

\section{Conclusions}

299 Community consultations were performed using in-person, print media, online, and mailed surveys 300 supported by clinical researchers. Survey respondents differed in some demographics from the general 301 public and did not perfectly reflect the target study population. In-person consultations at large public 302 events offered advantages in terms of cost and time commitment of researchers, while providing 303 respondent demographics closest to potential study community demographics. After two decades of 304 EFIC, improved guidelines for community consultation methodology should be developed and 305 discussed. Such guidelines could help both local IRBs and researchers with future EFIC trials. 306 307 


\section{Acknowledgements}

310 Maine: We acknowledge Barbara McCrum RN, Lauren Connolly RN, Adelene Hills BA, the MMC

311 Emergency Department Staff, Maine Red Claws Staff, and our patient and family participants in the 312 community consent process.

313 Arizona: We acknowledge Katie Mass, Ashley Larsen, and the University of Arizona College of 314 Medicine REACT (Resuscitation Education and CPR Training) Group, without whose services our 315 community consultation process would not have been possible.

\section{Funding}

319 This work was funded by the Arizona Biomedical Research Commission through an Arizona

320 Biomedical Investigator Award (AZ BIG) entitled, “A Pilot Randomized Clinical Trial of Early

321 Coronary Angiography versus No Early Angiography for Post-Cardiac Arrest Patients without ECG 322 ST Segment Elevation” (ADHS14-082985). 


\section{References}

327 1. General Requirements for Informed Consent. 21 C.F.R. $§ 50.20$ (1981, amended 1999).

328 2. Mozaffarian D, Benjamin EJ, Go AS, et al. Heart Disease and Stroke Statistics-2016 Update: A 329 Report From the American Heart Association. Circulation. 2016;133:e38-60.

330 3. Centers for Disease Control and Prevention. A Public Health Action Plan to Prevent Heart 331 Disease and Stroke. https://www.cdc.gov/dhdsp/action_plan/pdfs/action_plan_full.pdf. (2000, $332 \quad$ updated 2008).

333 4. Gale SC, Shafi S, Dombrovskiy VY, el al. The public health burden of emergency general 334 surgery in the United States: A 10-year analysis of the Nationwide Inpatient Sample--2001 to 2010. J Trauma Acute Care Surg. 2014;77:202-208.

5. FDA (2013). Guidance for Institutional Review Boards, Clinical Investigators, and Sponsors: Exception from Informed Consent Requirements for Emergency Research. Silver Spring, MD: Food and Drug Administration, Center for Drug Evaluation and Research: Division of Drug Information.

6. Fehr AE, Pentz, RD, Dickert NW. Learning from Experience: A Systematic Review of Community Consultation Acceptance Data. Ann Emerg Med. 2015;65:162-171.

7. Dickert NW, Govindarajan P, Harney D, et al. Community Consultation for Prehospital $2014 ; 18: 274-281$

345 8. Stephens SW, Williams C, Gray R, Kerby JD, Wang HE. Preliminary Experience with Social Media for Community Consultation and Public Disclosure in Exception from Informed Consent Trials. Circulation. 2013;128:268-270. 
348 9. Davis DP, Garberson LA, Andrusiek DL, et al. A Descriptive Analysis of Emergency Medical 349 Service Systems Participating in the Resuscitation Outcomes Consortium (ROC) Network. $350 \quad$ Prehosp Emerg Care. 2007;11:369-382.

351 10. Nelson MJ, Delorio NM, Schmidt TA, et al. Local Media Influence on Opting Out from an 352 Exception from Informed Consent Trial. Ann Emerg Med. 2010;55:1-8.

353 11. Bulger EM, Schmidt TA, Cook AJ, et al. The Random Digit Dialing Survey as a Tool for 354

12. Blumberg S, Luke JV. Wireless Substitution: Early Release of Estimates From the National Community Consultation for Research Involving the Emergency Medicine Exception from Informed Consent. Ann Emerg Med. 2009;53:341-350.

14. McComas KA, Besley JC, Trumbo CW. Why Citizens Do and Do Not Attend Public Meetings about Local Cancer Cluster Investigations. Policy Studies Journal. 2006;34:671-698

15. Sims CA, Isserman JA, Holena D, et al. Exception from Informed Consent for Emergency

366 16. Maher Z, Grill EK, Smith BP, Sims CA. Does Proximity to Violence Negatively Influence Attitudes Toward Exception from Informed Consent in Emergency Research? J Trauma Acute 368 Care Surg. 2015;79:364-371. 
369 17. Dickert NW, Mah VA, Baren JM, et al. Enrollment in Research under Exception from

Informed Consent: The Patients' Experiences in Emergency Research (PEER) Study. Resuscitation. 2013;84:1416-1421.

18. Wilets I, O'Rourke M, Nassisi D et al. How Patients and Visitors to an Urban Emergency Department View Clinical Research. Acad Emerg Med. 2003;10:1081-1085.

19. Goldstein JN, Espinola JA, Fisher J, Pallin DJ, Camargo CA. Public Opinion of a Stroke Trial Using Exception from Informed Consent. Int J Emerg Med. 2010;3:385-89.

20. Abboud PA, Heard KA, Al-Marshad AA, Lowenstein SR. What Determines Whether Patients Are Willing to Participate in Resuscitation Studies Requiring Exception from Informed Consent? J Med Ethics. 2006;32:468-472.

21. McClure KB, Delorio NM, Gunnels MD et al. Attitudes of Emergency Department Patients and Visitors Regarding Emergency Exception from Informed Consent in Resuscitation Research, Community Consultation, and Public Notification. Acad Emerg Med. 2003;10:352359.

22. Biros MH, Sargent C, Miller K. Community Attitudes Towards Emergency Research and Exception from Informed Consent. Resuscitation. 2009;80:1382-1387.

23. Longfield JN, Morris MJ, Moran KA et al. Community Meetings for Emergency Research Community Consultation. Crit Care Med. 2008;36:731-736.

24. Nelson M, Schmidt TA, DeIorio NM et al. Community Consultation Methods in a Study Using Exception to Informed Consent. Prehosp Emerg Care. 2008;12:417-25.

25. Smithline HA, Gerstle ML. Waiver of Informed Consent: A Survey of Emergency Medicine Patients. Am J Emerg Med. 1998;16:90-91. 
391 26. Contant C, McCullough LB, Mangus L et al. Community Consultation in Emergency

392 Research. Crit Care Med. 2006;34:2049-2052.

393 27. Nelson MJ, DeIorio N, Schmidt TA et al. Why Persons Choose to Opt Out of an Exception

394 from Informed Consent Cardiac Arrest Trial. Resuscitation. 2013;84:825-830.

395 28. Stephens SW, Williams C, Gray R et al. Utilizing Social Media for Community Consultation

396 and Public Disclosure in Exception from Informed Consent Trials. J Trauma Acute Care Surg.

$397 \quad 2016 ; 80: 1005-1009$.

398 29. Biros MH, Runge JW, Lewis R, Doherty C. Emergency Medicine and the Development of the

$399 \quad$ Food and Drug Administration's Final Rule on Informed Consent and Waiver of Informed

400 Consent in Emergency Research Circumstances. Acad Emerg Med. 1998;5:359-368.

401 30. Flynn G. Community Consultation for Emergency Exception to Informed Consent: How Much

402 is Enough? Ann Emerg Med. 2008;51:416-419.

403 31. Lewis RJ. Community Consultation by Randomly Reaching Out to the Community. Ann $404 \quad$ Emerg Med. 2009;53:351-353.

405 32. Robinson EJ, Kerr CE, Stevens AJ, et al. Lay public's understanding of equipoise and 406 randomisation in randomised controlled trials. Health Technol Assess. 2005;9:1-192, iii-iv.

407

408

409

410 


\section{$411 \quad$ Figure Legends}

412 Figure 1.

413

Summation of community and personal acceptances by modality in Maine and Arizona.

414

415 Figure 2.

416

Community and personal acceptance of EFIC stratified by age or education in Maine and

417 Arizona. $\mathrm{CA}=$ community acceptance, $\mathrm{PA}=$ personal acceptance

418 